\title{
La Unión Europea y el Mercosur: Dos bloques regionales buscan fortalecer sus vínculos
}

\author{
Amalia Stuhldreher
}

El ritmo dilatorio de liberalización comercial a nivel multilateral afecta directamente las negociaciones entre la Unión Europea y el MERCOSUR, que apuntan a una asociación interregional de carácter político y económico. Sin embargo, paralelamente a la definición de los esquemas de negociación acerca de las barreras no tarifarias al comercio con su partner sudamericano, Europa confirma su compromiso con el MERCOSUR a partir de un intento de fortalecimiento del diálogo político. Para ambas partes el desafío consiste en aprovechar los márgenes de maniobra, que son acotados, para hacer uso de interesantes espacios para profundizar la cooperación entre los socios.

\section{Negociaciones en pos de una asociación interregional}

La última Cumbre de los Presidentes del MERCOSUR los días 29 y 30 de junio pasados, durante la cual Argentina le transfirió a Brasil la presidencia pro tempore del Consejo de dicho bloque, contó con la presencia de un invitado inusual. El Alto Representante de la Unión Europea (UE), Javier Solanas, eligió dicha ocasión como momento propicio para manifestar el decidido interés de los europeos en reimpulsar las relaciones con la aún imperfecta unión aduanera del Cono Sur, en especial a través del fortalecimiento del diálogo político. La visita de Solanas invita a reflexionar acerca del nivel de concientización europeo respecto a la necesidad de emitir una clara señal que confirme su compromiso con el MERCOSUR.

Cabe recordar que la redefinición del parámetro de vinculación entre la entonces Comunidad Europea y el MERCOSUR a principios de los años '90 se dio como consecuencia del decidido apoyo de los europeos al proyecto integrativo sudamericano, donde se destaca especialmente el Acuerdo de 
Cooperación Institucional de mayo de 1992. A través de la concreción del Acuerdo Marco de Cooperación de diciembre de 1995 se apuntó explícitamente a generar las condiciones para el establecimiento de una asociación interregional de carácter político y económico. En ese marco se llevó a cabo a fines de junio de 1999 la Cumbre de Río de Janeiro entre la UE y 33 pares de América Latina y el Caribe. La evaluación de dicho encuentro osciló entre postular que se había tratado de un acontecimiento "histórico" o en afirmar que en realidad el encuentro encarnaba la "cumbre de la hipocresía". ${ }^{1} \mathrm{Sin}$ embargo, en lo que respecta al acercamiento UE-MERCOSUR, se registraron algunos avances: Durante dicho encuentro los mandatarios de ambas partes, así como de Chile, (país asociado a la unión aduanera sudamericana desde 1996, que firmara en el mismo año en Florencia un acuerdo con la UE de características similares al vigente entre el MIERCOSUR y la UE), emitieron un Comunicado conjunto donde reafirmaron su intención de fortalecer los vínculos interregionales y acordaron negociaciones encaminadas a la liberalización bilateral, gradual y recíproca del comercio, con la inclusión explícita de todos los sectores y de conformidad con los lineamientos de la Organización Mundial del Comercio (OMC). Se decidió que a través de las reuniones del Consejo de Cooperación surgido del Acuerdo Marco de 1995 se comenzaría a negociar en noviembre de 1999 acerca de la estructura, la metodología y el calendario de las negociaciones. ${ }^{2}$ Se acordó asimismo que dichas conversaciones, que tendrían lugar en Bruselas, se referirían a barreras al comercio de carácter no tarifario. A más tardar el $1^{\circ}$ de julio del año 2001 deberían comenzar las negociaciones sobre la disminución de las barreras aduaneras. No se estableció una fecha precisa para la firma del acuerdo de libre comercio, aunque los Estados del MERCOSUR querían fijar el año 2005 para el cierre de las negociaciones. En definitiva se puso en evidencia la inevitabilidad del previo cierre de la Ronda del Milenio de carácter multilateral para poder avanzar decididamente en el logro de las metas de la asociación interregional.

\footnotetext{
- Véase respecto al balance de la Cumbre de Río: Bodemer, Klaus. "îl comienzo de una coincidencia estratégica? La primera Cumbre UE-América Latina/Caribe, en Río de laneiro", en: D+C Desarrollo y Cooperación. DSE. N s. Septiembre/octubre 1999. (Págs. 8•11), así como tamblén: Informe IRELA. "La Cumbre de Río: ¿Hacia una asociación estratégica?". 30 de julio de 1999.

2 Véase: "Comunicado Conjunto de Río de Janeiro", en: Informe especial de IRELA. "Las perspectivas de un Acuerdo de Libre Comercio UE-MERCOSUR y las opciones para la política de Estados Unidos." $1^{\circ}$ de noviembre de 1999. (Págs. 33-34).
} 
Los encuentros prosiguieron durante la primera semana del mes de abril pasado, cuando los representantes del MERCOSUR se reunieron en Buenos Aires con una misión de la UE con el objetivo de definir la metodología de las dilatadas negociaciones de liberalización del comercio mutuo. En un comienzo dichas reuniones fueron interpretadas como una señal de "deshielo" de las relaciones, en especial en el terreno político: En ese sentido fue significativo el número de los funcionarios de la delegación europea, encabezada por el Director de Relaciones Exteriores de la Comisión Europea, Guy Legras, quien secunda al Comisario británico Chris Patten. Las discusiones, de tenor eminentemente técnico, no permitieron empero modificar la decisión de la UE de esperar hasta julio del 2001 para empezar a discutir las cuestiones tarifarias. Aunque la prensa sudamericana destacó especialmente el hecho de que tampoco se lograron adelantos decisivos en el tema del comercio de productos agrícolas, ha de destacarse que por su carácter, las reuniones de abril no apuntaban a aspectos sustantivos sino más bien operativos. Más allá de eso, el tema agrícola en sí es un "rehén" de las negociaciones de la OMC en Ginebra. Los lineamientos para este sector se retrasan ademâs debido al fracaso de la reunión preparatoria de la Ronda del Milenio en Seattle en noviembre 1999. Con este telón de fondo, la ronda de negociaciones en Buenos Aires no estuvo exenta de tensión. Tras dos días de tratativas se conformaron tres grupos de trabajo que se abocarán a la desmantelación de las trabas no arancelarias al comercio. El primer grupo se dedicará al análisis del intercambio de bienes, el acceso a mercados y las normas de origen. Concretamente se apuntará a reducir las trabas sanitarias y fitosanitarias. El segundo grupo se concentrará en el intercambio de servicios, las trabas a la inversión y los sistemas de protección intelectual. Finalmente, el tercer grupo encarará las políticas de competencia, los sistemas de compras gubernamentales y de solución de controversias comerciales. Como expresara un funcionario de la representación de la Comisión de la UE en Buenos Aires, las noticias resultantes de este encuentro no eran particularmente "sexy" para los medios locales, lo cual tenía que ver en especial con expectativas desmedidas de lo que era posible acordar en una reunión de este tipo. Igualmente, al término del encuentro, la parte sudamericana volvió a insistir en que se debe avanzar en forma más decidida. En efecto, según su visión las dilaciones en las negociaciones con el MERCOSUR contrastan claramente con los adelantos efectivos de las tratativas de la UE con Sudăfrica y con México, que condujeron a una rápida 
concreción de acuerdos comerciales en escaso tiempo. Para los representantes deI MERCOSUR para lograr resultados similares, el presente proceso de negociación con la UE requiere en forma ineludible de un "breakthrough" en la cuestión agrícola. Por su parte, los europeos se mostraron satisfechos de haber sentado bases sólidas para encarar las negociaciones siguientes fuera del ámbito de los reflectores de la "gran política".

Más allá de ésto, y en coincidencia con la visita de los funcionarios de la UE, se destacó por otra parte la presencia en Buenos Aires del canciller francés Hubert Védrine: en sus encuentros con los funcionarios del gobierno argentino, Védrine concretó una serie de consultas políticas, en las que no excluyó el espinoso tema agrícola y que se verían complementadas por la visita del secretario de comercio francés, Francois Huwart, durante el mes de mayo. Por otra parte, en el momento en que se iniciaban las conversaciones UEMERCOSUR, el jefe de la delegación de la UE en la Argentina, Vittorino Allocco adelantó la posible firma de un acuerdo alimentario entre la UE con Argentina, Chile y Uruguay, que permitirían que las exportaciones agroalimentarias de estos países se vieran exhimidas de los controles sanitarios al ingresar al mercado comunitario europeo. Aunque dicho anuncio repercutió favorablemente en los ámbitos empresarios de los países sudamericanos, no se consideró que ésto constituya una solución al problema real que enfrentan los exportadores del Cono Sur, esto es la política de subsidios y de cupos vigente en la UE. Al respecto debe subrayarse, empero, que existen avances en la materia: paralelamente a la reducción de un $20 \%$ de los subsidios pagados a los productores europeos, se procedió a congelar hasta el año 2006 el gasto comunitario agrícola en 40.5000 millones de Euros. ${ }^{3}$

Durante la tercera semana de junio las partes volvieron a reunirse en Bruselas, donde se llevó a cabo la segunda ronda de negociaciones interregionales. Los magros resultados terminaron confirmando el ánimo alicaído del mes de abril, especialmente entre los funcionarios argentinos, que acusan a la UE de recurrir a maniobras dilatorias. En lo que hace a la visita de Solanas de fines de junio, se destaca primordialmente la intención europea de acentuar la cooperación política con su partner sudamericano. Concretamente el funcionario apuntó a la transferencia de experiencias de la UE susceptibles de ser aplicadas al MERCOSUR: aspectos salientes de esta transferencia de know-

3 Véase: Diario La Naciôn. 1/4/2000. 
how integracionista están dados por la coordinación intensificada en materia de política exterior, en la gestión de crisis y en los aspectos militares. Este aporte podría resultar especialmente relevante: Observadores, analistas y actores de ambas partes confirman y señalan la necesidad impostergable de que el MERCOSUR fortalezca sus mecanismos de coordinación de posiciones comunes. En esta discusión se destaca la necesidad de un salto cualitativo del bloque que apunte a la incorporación de mecanismos supranacionales en la toma de decisiones. Por su parte, Solanas destacó en forma especial la dimensión simbólica de su visita, que apunta a la reafirmación de los compromisos políticos de la UE ya asumidos con la región.

\section{El horizonte actual de la relación EU-MERCOSUR}

Ante las tendencias imperantes a nivel global cabe la pregunta concreta acerca de la relevancia estratégica de la posible profundización de la relación interregional. Excluyendo posicionamientos voluntaristas, es evidente que la concreción de una asociación se verá directamente afectada por la evolución respectiva de ambos bloques. La agenda interna del MERCOSUR presenta desafíos institucionales y estructurales significativos, exhacerbados por la devaluación del real brasilero a comienzos de 1999. Aunque en la actualidad el MERCOSUR todavía no ha logrado constituir una unión aduanera plena, ${ }^{4}$ desde comienzos de este año y a partir de los cambios de gobiernos en sus países miembros, así como en Chile, se registra una clara voluntad política de impulsar y ampliar el emprendimiento regional tras el impasse de los últimos dos años. ${ }^{5}$ Este compromiso quedó claramente confirmado con el anuncio del presidente chileno Ricardo Lagos del mes de julio, cuando anunciara que su país formulará el pedido de ingreso formal al MERCOSUR en diciembre

\footnotetext{
4 Un aspecto que ilustra dicho estadio es la reciente decisión del gobierno argentino de prolongar el aran. cel diferencial para el azúcar, que debía eliminarse en el 2001, para proteger a las economías regionales del Noroeste del país. Asimismo existen desacuerdos por ejemplo con respecto a la definición de un nuevo régimen automotor, tras que Uruguay se negara a adherir al acuerdo alcanzado a fines de junio en Buenos Aires por los otros miembros del bloque.

s Una señal positiva en ese sentido es la Intención manifiesta de reducir el arancel externo común (AEC), que en promedio asciende al $14 \%$, para impulsar el acercamiento de Chile al bloque. Dicho pais posee una estructura arancelaria unitaria que ronda en el $9 \%$, que debe disminuir progresivamente hasta llegar al $6 \%$ en el 2003. En virtud de ello se le concedió a Chile un margen de maniobra relativamente alto para la concepción de su política comercial exterior al momento de asociarse al MERCOSUR.
} 
próximo. Por su parte, y como se repite incansablemente, la UE también enfrenta los enormes desafíos respecto a su proceso de ampliación y profundización.

Pese a todo ello, si se hace una evaluación a mediano y largo plazo, se identifica un gran potencial para la constitución de una asociación interregional, cuya concreción de todas formas no es inminente: La UE ocupa el primer lugar en el comercio del MERCOSUR, acumulando un crecimiento entre 1990 a 1998 del orden del 375\%, donde la participación comunitaria dentro de las importaciones totales del MIERCOSUR se elevó del 14,4\% al 21,6\%, destacándose sectores como maquinaria y equipos de transporte, sustancias químicas y manufacturas básicas. A partir de 1995 se invirtió definitivamente la tendencia superavitaria tradicional a favor del MERCOSUR, de allí la insistencia sudamericana de mejorar su acceso al mercado comunitario. Paralelamente, desde 1996 la UE se convirtió en el primer inversor en el bloque sudamericano, superando así a los Estados Unidos y al Asia. Así en el año 1998 los europeos efectuaron el 46,2\% de las inversiones externas directas, frente al $27,2 \%$ de procedencia estadounidense. ${ }^{6}$ Observadores del proceso ponderan los beneficios de esta asociación para los países del MERCOSUR, como la gestación de un contrapeso interesante frente a los Estados Unidos, así como una mayor diversificación de las relaciones comerciales, la transparencia de precios otorgada por la existencia de una moneda única europea para los exportadores e inversores del MIERCOSUR interesados en proyectarse al ámbito europeo, o el mejor acceso al mercado europeo para productos agropecuarios y pesqueros procedentes de Sudamérica. Por su parte, la UE espera que el establecimiento de un vínculo especial con el Cono Sur le permita mantener y afianzar su significativa posición en la subregión frente al posible peligro de una hegemonía total por parte de Estados Unidos en caso de concretarse el acuerdo del ALCA (Asociación de Libre Comercio Americana), descle Alaska a Tierra del Fuego. De acuerdo a un estudio de la Comisión de la UE, dicho bloque se beneficiaría en mayor medida de la concreción de un ALC (Acuerdo de Libre Comercio) con el MERCOSUR, que a la inversa: en ese sentido las ganancias adicionales alcanzarían el orden de 6.200 millones de dólares para la UE y de 5.100 millones para el MERCOSUR. A esto debieran sumarse los efectos positivos sobre los flujos de inversión. ${ }^{7}$

\footnotetext{
"Véase: Informe de IRELA. 1 de Noviembre de 1999. Op. citat, 11-12, asi como Informe de IRELA. "Preparando la Asociación UE-MERCOSUR. Beneficios y obstáculos". 20 de julio de 1998. Pág. 5.

7 Véase respecto al mencionado estudio: Informe de IRELA. 1 de noviembre de 1999. Op. citat. Pág. 15.
} 


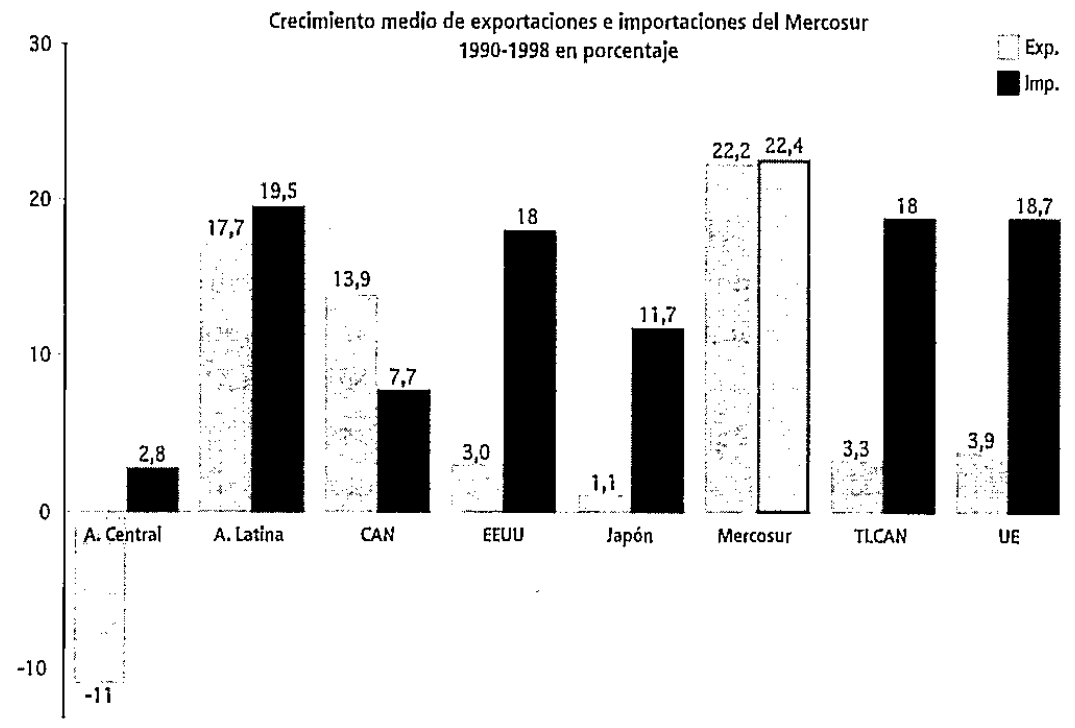

Fuente: Elaboración propia a partir de datos del LMF, Direction of Trade Statistics Yearbook 1998. Washington DC, 1998; LMF, Direction of Trade Statistics Quaterly, Washington DC, Jine 1999 y cálculos de IRELA, citados en: Informe IRELA. "LAs perspectivas de un acuerdo de libre comercio UE-MERCOSUR y las opciones para la polírica de EE UU". I de noviembre de 1999. (Pág. 38).

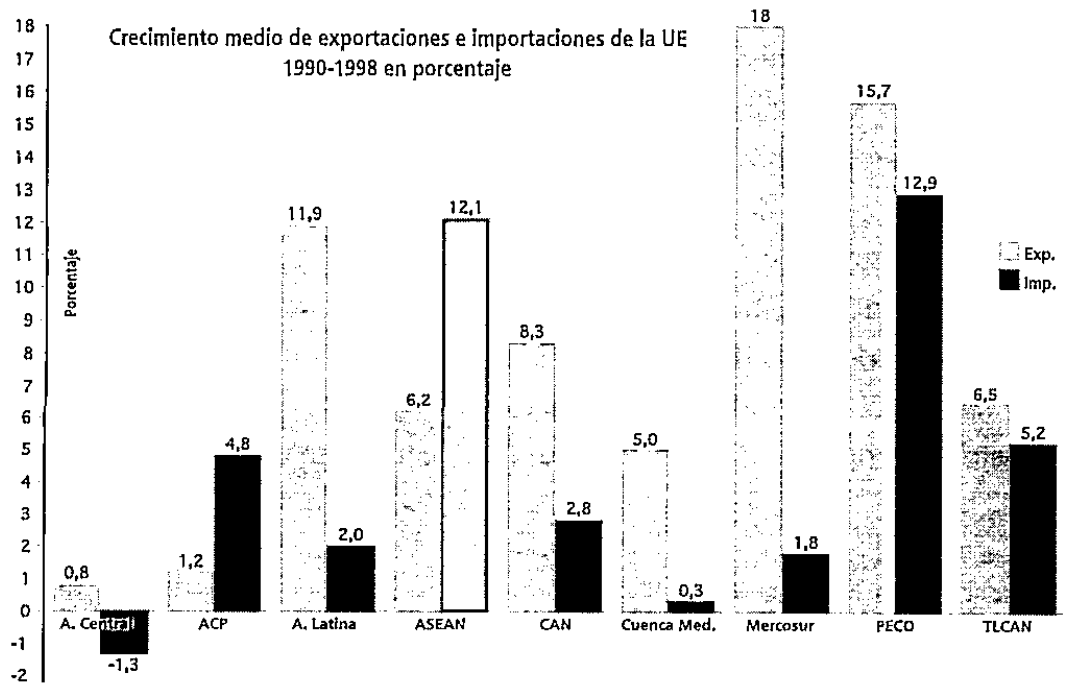

Fuente: Elaboración propia a partir de datos del MMF, Direction of Trade Statistics Yearbook 1998. Washinglon DC, 1998; IMF, Direction of Trade Statistics Quaterly, Washington DC, June 1999 y cálculos de IRELA, citados en: Informe IRELA. "Las perspectivas de un acuerdo de libre comercio UE-MERCOSUR y las opciones para la politica de EE UU". I de noviembre de 1999. (Pág. 40). 
Pese a todo, las dificultades a nivel comercial no son susceptibles de ser superadas rápidamente. Mientras que la UE tiende a proteger su sector agrícola y pesquero, el MERCOSUR se torna proteccionista en cuestiones como liberalización del sector servicios, bienes de capital, telecomunicaciones, y productos informáticos. Un acápite que merece especial atención es el automotriz, por su dinamismo y por la gestación de una alianza estratégica con la contraparte europea, la que asimismo muestra un marcado interés europeo por el sector servicios y compras gubernamentales en el área MERCOSUR. ${ }^{8}$

No puede olvidarse aquí que el MERCOSUR no representa una prioridad absoluta para la UE. A esto se suma el hecho de que el modelo elegido de asociación con el MERCOSUR representa para los europeos un novum. En forma genérica la base jurídica de la asociación está representada por los Artículos131 a 136, así como el Artículo 238 del Tratado de la UE, donde no se define exactamente dicho instrumento. En el Acto Jurídico preparatorio en el que la Comisión autoriza la firma del acuerdo interregional con el MERCOSUR, se hace referencia explícita al Art. 113 (Política Comercial Común), al Art. 130 y (Acuerdos de cooperación) y al Art. 228 (Acuerdos con terceros Estados y organizaciones). ${ }^{9}$ Concretamente se trata aquí de un acuerdo mixto, es decir que tanto la UE en su conjunto, así como también sus países miembros a nivel individual se convierten en signatarios y partes del acuerdo, debido a que no sólo se ve afectada la competencia de la UE en materia de política comercial, sino también las respectivas competencias nacionales. Esto implica la necesidad de ratificaciones parlamentarias por parte de cada Estado, con los retrasos consiguientes para el proceso en su totalidad. Por otra parte, el mencionado modelo posee un bajo nivel de concretización, ya que la meta general definida de generar las condiciones para una asociación interregional con objetivos de orden político y económico evita explícitamente la mención de objetivos directos como el establecimiento de una zona de libre

\footnotetext{
${ }^{8}$ Véase al respecto: Informe de IRELA. "MERCOSUR-Unión Europea: Dinámicas y perspectivas de una asociación creciente". 19 de febrero de 1999.

- Véase: Acto Jurídico preparatorio de la Comisión Vorbereitende Rechtsakte. Kommission. Vorschlag für einen Beschluß des Rates über den Abschluß des interregionalen Rahmenabkommens über die Zusammenarbeit zwischen der Europäischen Gemeinschaft und ihren Mitgliedstaaten einerseits und dem Gemeinsamen Markt des Südens und seinen Mitgliedstaaten andereseits, en: Amtsblatt der Europäischen Gemeinschaften: Mitteilungen und Bekanntmachungen. Luxemburg. 39. (19. Januar 1996). C 14. (Pâg. 3). 'v Véase: Utrich Wehner. "Der MERCOSUR. Rechtsfragen und Funktionsfähigkeit eines neuen Integrationsprojektes und die Effolgsaussichten der interregionalen Kooperation mit der EU". Nomos Verlagsgesellschaft. Baden-Baden. 1999. Păgs. 192-194 asi como Págs. 212-213 y Pág. 221.
} 
comercio en el Acuerdo de $1995 . .^{10}$ Sin embargo, las negociaciones de la UE con un grupo de países como el MERCOSUR representa claramente un intento de "racionalizar" sus relaciones de cooperación hacia otras regiones.

\section{Las perspectivas de una asociación estratégica}

A la hora de evaluar las perspectivas de la relación entre la UE y el MERCOSUR con frecuencia se presenta a la misma como una necesidad ineludible de cara al futuro, ya que pese a las claras asimetrías vigentes no existen en el mundo contemporáneo otras dos regiones que mantengan relaciones tan estrechas y diversas en los ámbitos político, económico y cultural. Reiteradamente se invoca el elemento histórico, cultural y axiológico común como base que permitiera concretar en un futuro cercano un accionar conjunto. Sin embargo, dicha base enfrenta claras limitaciones en un contexto global en donde las alianzas posibles son de signo cambiante. Que dicho acervo común podría resultar insuficiente para generar una cooperación sólida en el futuro y que no es susceptible de ser utilizado y racionalizado a ultranza a manera de mecanismo "compensatorio" de avances concretos, ha sido ya puesto de manifiesto por algunos observadores del proceso." Por otra parte, un examen realista no puede obviar el hecho que no es posible esperar grandes progresos en un futuro próximo para el establecimiento de una "relación especial" entre ambos bloques: el espacio de maniobra de la UE frente a Latinoamérica en general y al MERCOSUR en particular se ve acotado debido a otros compromisos económicos y de política exterior asumidos por el bloque europeo. ¿Cuál es entonces el margen de acción existente?

La evidencia empírica a la que se hizo referencia indica que éste no pareciera ser demasiado amplio. En algunos círculos se formula la pregunta si todos los últimos movimientos de la UE no se reducen en definitiva a dilaciones tácticas. Europa debiera evitar que se fortalezca la impresión ya existente en ese sentido, de que la UE carece de una verdadera estrategia de fondo con

"Véase al respecto: Informe IRELA. "Las relaciones entre Europa y América Latina: hacia una agenda birregional para el Siglo XXI". Preparado para la Cumbre de Jefes de Estado y de Gobierno de la Unión Europea, América Latina y el Caribe a instancias de la Presidencia de la Unión Europea. Río de Janeiro, 2829 de junio de 1999. 
lineamientos claros. Ante la sombra de las dificultades para avanzar en la Ronda del Milenio y dado que el "estímulo negativo" procedente de la posible concreción del ALCA se desvanece en este año electoral para EE UU, la UE se retrotrae en una "diplomacia de demostración", ${ }^{2}$ esperando que se despejen las otras incógnitas de la ecuación. El riesgo implícito en esta maniobra es la pérdida del capital político acumulado en Sudamérica, así como la eventual reducción de su participación en dicho mercado. En vista del precedente de las consecuencias que tuvo el ingreso de México al TLCAN (Tratado de Libre Comercio de América del Norte) para sus intercambios con Europa, la UE no puede ignorar simplemente los riesgos de desviación de comercio e inversiones en el Cono Sur en caso de la firma del ALCA. Aún teniendo presente lo espinoso de los temas de la agenda bilateral y frente a la futura reunión que se realizará entre octubre y noviembre en Brasil, el tono dilatorio debiera modificarse para evitar un estancamiento definitivo. Dado que los sudamericanos no consideran su relacionamiento con la UE como una alternativa excluyente del proceso del AL.CA, sino que privilegian una interpretación que acentúa la complementaridad de ambos emprendimientos, un eventual fracaso en el relanzamiento del eje UE-MERCOSUR puede llevar a ambas partes a revisar las prioridades, a la introspección o el reemplazo de los socios seleccionados. Cada decisión en uno u otro sentido implica costos de oportunidad que debieran ser cuidadosamente examinados. Una visión cortoplacista no debiera oscurecer el potencial implícito en la relación UE-MERCOSUR: Si se tiene en cuenta que en la Cumbre de Río de 1999 la mayoría de los acuerdos se alcanzó en temas globales, enmarcados en los regímenes internacionales, resulta innegable que las coincidencias no son meras casualidades secundarias. Dicha base consensual de la cual ya se dispone debería ser estratégicamente potenciada para generar una coordinación mancomunada que permita ejercer un peso decisivo en la gestación conjunta de la arquitectura de las relaciones internacionales en el siglo entrante, aunque tampoco aquí caben las esperanzas desmedidas.

En lo que hace a las cuestiones comerciales, la discusión académica reconoce ya hace tiempo que los procesos de liberalización comercial a nivel multilateral y los avances del así llamado" nuevo regionalismo" de carácter abierto no tienen por qué resultar contradictorios entre sí. Las iniciativas regionales de los últimos años (entre ellas el MERCOSUR) tienden más bien a conformar los así llamados "building blocks", a la manera de ladrillos cons- 
titutivos de una economía mundial integrada, y no en "stumbling blocks" que dificultan dicha evolución. ${ }^{13}$ El nuevo regionalismo en América Latina es concebido incluso como una posible solución para revigorizar y actualizar el sistema multilateral. Especialmente significativo es su aporte a la integración del Norte y el Sur en una forma que supera ampliamente los logros del GATT (General Agreement on Tariffs and Trade). ${ }^{14}$ A partir de ello es donde justamente la relación UE-MERCOSUR así como las negociaciones en tomo del ALCA adquieren una connotación que supera los límites de los respectivos procesos en sí mismos: Ante la incertidumbre que impera en el seno de la OMC a partir del dificultoso avance de las negociaciones sobre agricultura ${ }^{15}$ y servicios, toda coordinación de posturas divergentes que se logre en los otros niveles puede convertirse en un input positivo susceptible de ser recogido en las tratativas multilaterales. Que esto constituye un ejercicio sumamente dificultoso, está fuera de toda discusión. Sin embargo, pese a las actitudes defensivas y proteccionistas presentes en cada lado, la existencia por otra parte de sectores dirigentes que identifican claramente la chance que implica un vínculo económico profundizado, permite algún nivel de optimismo de cara a la década que recién comienza. Dada la inevitable existencia del mutuo condicionamiento de los procesos, los esfuerzos debieran apuntar a evitar el estancamiento que puede generarse a partir de una interdependencia negativa entre los diferentes niveles de negociación.

12 Véase: Informe de IRELA. 30 de julio de 1999. Op. citat. Pág. 4.

${ }^{13}$ Véase al respecto: Lawrence, Robert. "Emerging Regional Arrangements: Building Blocks or Stumbling Blocks?", en: Frieden, Jeffry / Lake, David (Edit.) "Intenational Economy. Perspectives on Global Power and Wealth". Third Edition Routledge. London. New York. 1997. (Pags. 407-415). Resulta interesante comprobar que los decisores involucrados en el proceso UE-MERCOSUR analizado en este artículo coinciden ampliamente con las aseveraciones provenientes de la academia.

4 Véase: Tussie, Diana. "In the whirlwind of globalization and multilateralism. The case of emerging regionalism in Latin America", en: Coleman, William/ Underhill, Geoffrey (Edit). "Regionalism \& Global Economic Integration. Europe, Asia and the Americas". Routledge. London. New York, 1998. (Págs. 81-96).

is Las propuestas que el 30 de junio fueron dadas a conocer en Ginebra por los Estados Unidos, la UE y el Grupo Cairns reafirmaron la impresión de que la liberalización en este sector tenderá a dilatarse ante la notoria incompatibilidad de las posiciones. 